\title{
Resilience in Industry 4.0 Digital Infrastructures and Platforms
}

\author{
Daniel RIBEIRO ${ }^{\mathrm{a}, \mathrm{b}, 1}$, António ALMEIDA ${ }^{\mathrm{a}, \mathrm{b}}$, Américo AZEVEDO ${ }^{\mathrm{a}, \mathrm{b}}$ and Filipe \\ FERREIRA $^{\mathrm{b}}$ \\ ${ }^{a}$ Faculdade de Engenharia, University of Porto, 4200-465 Porto, Portugal \\ ${ }^{\mathrm{b}}$ INESC TEC - Institute for Systems and Computer Engineering, Technology and \\ Science, 4200-465 Porto, Portugal
}

\begin{abstract}
We live in a world where companies are shifting to the industry 4.0 paradigm. One of the pillars of Industry 4.0 is the digitalization of physical assets and manufacturing processes, moving toward the Cyber-Physical Production Systems concept (CPPS). In these systems, every component of the production process - machines, tools, workstations, etc. - is equipped with sensors, possesses information about itself, and can interact with each other, allowing the production of smaller batches at lower prices and increase product customization through adaptative processes. Consequently, companies are evolving their information systems to have more visibility and control over their production systems. This change increases both the production system's agility and its vulnerability to communication and information related disruptions. Hence, companies that adhere to Industry 4.0 enabling technologies must adopt new methodologies and tools to become aware of the new risks that arise by the introduction of new digital platforms, their impacts in the production systems, and how they may react to remain resilient. In this paper, disruption events and adequate mitigation strategies are analysed, modelled, and simulated as part of a methodology designed to measure the impacts of disruptive events on the production system.
\end{abstract}

Keywords. Industry 4.0, Cyber-Physical Production System (CPPS), disruption management, hybrid simulation.

\section{Introduction}

Industry 4.0 consists of a paradigm shift from automated production to an intelligent production concept where all physical assets such as products, components, workstations, and machines possess individual information about themselves and are part of a network with communication interfaces, where all participants can interact with each other using technologies such as the Internet of Things. These assets are now called Cyber-Physical Systems (CPS), and the manufacturing system itself a Cyber-Physical Production System (CPPS). CPPSs are highly flexible systems that allow small batches to be produced at lower prices and increase product customization through adaptive production processes [1].

Consequently, companies are evolving their information systems to have more visibility and control over their production systems. As an example, companies are

\footnotetext{
${ }^{1}$ Corresponding author, E-mail: daniel.s.ribeiro@inesctec.pt.
} 
investing in cloud-based manufacturing execution systems (MES). Although this evolution allows the production to become more agile, it also increases the dependency on digital manufacturing platforms and vulnerability to new types of information and communication disruptions [2]. In this sense, these disruptions caused by missing information or communication failures are now the new production system's bottleneck, which requires the development of new tools to manage and control them.

This paper proposes the use of simulation as a tool to allow decision-makers to predict possible disruptions, their impacts on the production processes and to create a database with effective countermeasures. Thus, this paper aims to answer the following questions: (1) What are the main process disruptions in CPPSs? (2) How can the impacts of process disruptions in CPPSs be evaluated using simulated-based approaches? (3) How can we quantify and improve the resilience of CPPSs? Using action research methodology, disruption events and adequate mitigation strategies are analysed, modelled, and simulated as part of a methodology designed to measure the impacts of disruptive events on the production system. A use case study will be performed to test and demonstrate the application of the methodology.

\section{Literature Review}

\subsection{Disruptions}

Generally, a process disruption is an unwanted event that leads to a non-executable process during the execution of the current operation, where the deviation from the plan is sufficiently large that the plan has to be changed substantially [3]. In this definition, disruption is the event that leads to the deviation, but not the deviation itself as seen in [4]. As such, a process deviation is defined as the effect of a disruption event.

Disruptions include both disturbances and failures since both can cause the process to be disrupted. A production disturbance is an unplanned or undesirable state or function of the system and can occur in different parts of a manufacturing system [5], due to missing components, blocked manufacturing stations, missing workforces, manufacturing stations, resources, and workers. Failures are described as the nonfunctional state of a system. Because of the increasing use of communication technologies in cyber-physical production systems and the importance of information transparency, disturbances can also be caused by missing required manufacturing information or a failure of the communication interfaces [2]. Failures in manufacturing systems may be caused by the missing workforce, mechanical breakdowns, or quality problems [6].

The lifecycle of process disruptions is divided into four phases [7]. The first phase is the detection phase. This is the time between the occurrence of the disrupting event from a normal operating state and the time the process deviation is noticed. The second phase is the analysis phase that comprises the diagnose and analysis of the causes of the disruptions. The third is the development phase, where the countermeasures to solve the process deviations are identified and tested. The fourth phase is the implementation phase where the solution previously defined is implemented and the system should return to its normal operating state. 


\subsection{Resilience}

Resilience describes the system's behaviour when a disrupting event occurs during runtime. The resilience of a production system can be measured by the ability of a production system to withstand disruptions through maintaining functions and structures, reducing the time in the disruptive state, and responding to disruptive events [8]. In this context, the concept of resilience incorporates both the principles of robustness and agility.

Robustness describes the ability of the system to cope with minor disruptions without adaptations. It is the feature of a system that allows it to resist change or external factors, maintaining its stability [9] and continuously providing the desired output [10]. Typically, robustness refers to a constructive approach that prevents uncertainty from limiting the functionality of production processes, giving resistance to anticipated changes [11]. On the other hand, agility describes the ability of the system to regain its original state by adapting to changes caused by severe disruptions. The main advantage of an agile system is that it converts quickly and smoothly without predefined adaptation plans, meaning that not all potential disturbances need to be known since alternative response actions will take place soon after disturbances occur [12].

\subsection{Simulation-based disruption management systems}

As process disruptions have a negative impact on the performance of a manufacturing system, reducing the effect of disruptions is the most important step to achieve resilience in the affected processes. Disruption management aims to reduce the overall time of the system in disrupted mode [7], which can be achieved by an effective decision-making process for developing countermeasures [13].

As seen in [2], [5], [7], a disruption management methodology can be divided in four stages (Figure 1). In the first stage are identified the main causes for process deviation in the manufacturing system, and for each cause, possible response actions are listed in the second stage. In the last stages, the response actions are simulated and the gains in resilience of the system are measured so that the best action can be identified.

Disruptions are bound to happen and to better respond to them, they need to be organized into categories. In the context of Industry 4.0, disruptions can be categorized according to the source of the event: products, human resources, production equipment, communication, and information [6], [7], [14].

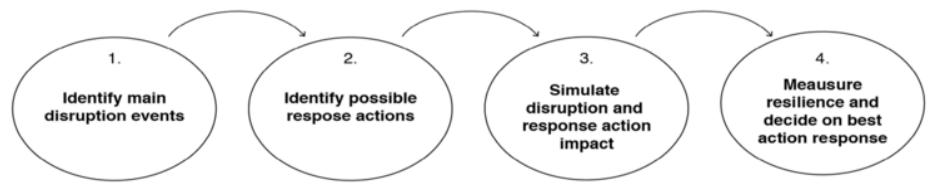

Figure 1. Stages of a disruption management methodology.

In [5], it is proposed that information about disruptions should be obtained from a manual logbook and interviews with the personnel. This way, specific disruptions from a particular production facility can be listed, thus allowing effective countermeasures to be studied and applied. The possible scenarios, upon a disruptive event, are bounded by the manufacturing centre limitations. So, it would be difficult to implement a general countermeasures database. Thus, the importance of gathering knowledge within the facility. 
The response actions for a given disruption event are simulated to illustrate their impact on the production processes. The impact will be measured using key performance indicators (KPIs), which in turn are used to compare the different scenarios. For measuring resilience, indicators such as production loss, throughput settling time, total under production time [15], and overall equipment efficiency (OEE) are widely used. For a general-purpose approach, state variables can also be mapped into the performance space [16].

\section{Concept Proposal}

The research's purpose is to create a solution that allows companies to assess the impact of disruptions in production systems and the support information systems for industrial processes. As introduced before, new applications of cyber-physical systems are coming to use, companies are adopting technologies such as the Internet of Things (IoT) and Cloud-based manufacturing, which lead to new types of disruption events. Disruptions that occur in a particular system may have impacts in the others and that is what the solution seeks to answer with the use of simulation tools.

The methodology is in line a disruption management system and will follow the four steps discussed in the previous chapter. First disruptions are categorized according to the source (Methods, Machine, Material, Man power and Milieu) [2] and location (production, logistics, supplier, production control, and others). Then, possible scenarios are gathered and simulated. Due to its wide use in the industry, the key performance indicator to be used for comparison is the OEE.

We hypothesize that a hybrid simulation-based solution can carry out the defined objectives. Hybrid simulations refers to the modelling approach that combines multiple simulation methods. In this case, agent-based simulation to model the different systems as agents, discrete event simulation (DES) to model the processes, and system dynamics (SD) for the production (production rate/time).

\section{Case Study Analysis}

The selected case study is a Portuguese company in the cork industry. This company has been increasing the level of digitization of its factories with the introduction of hardware to collect information and data from the shop floor and the installation of a manufacturing execution system (MES) for the management of its production processes. Despite the advantages of this digital transition, the company is concerned with the dependence on the digital infrastructure that has been set up and its ability to stay resilient in the event of disruptions.

With their concerns in mind, the methodology previously described was applied. In the interest of time, a single production station was implemented consisting of a double belt press responsible to produce cork sheet rolls. The production system receives orders from the MES, stores them in queue and proceeds with the scheduled production cycle. During a process, the cyber-physical production system is assumed to possess the ability to self-diagnose, that is, if a disruption event occurs, it can signal the MES about the event. To demonstrate the concept, the communication failure between MES and production system disruption will be introduced in the system. 
In this case the production cannot proceed without storing data in the MES database, so two different scenarios are originated: (1) Production finishes current order and waits for the connection to be re-established, or (2) the production data is stored in a buffer within the facility and the orders follow the normal scheduling. The MES database is later updated. To measure the impact of the disruption in the system, the reference state, where no disruption occurs, will also be simulated.

\section{Results and Discussion}

The simulation was executed in the hybrid simulation software Anylogic. The simulation corresponds to a full working week since the station does not stop until all the scheduled orders are complete. The disruption will have a cyclic reoccurrence time of 5 hours, and two different durations, one longer than the other.

Table 1. Simulation results for the reference state and disruption scenarios, with a short and long recovery time

\begin{tabular}{ccccc}
\hline Reference state & \multicolumn{2}{c}{ Long disruption } & \multicolumn{2}{c}{ Short disruption } \\
\hline & Scenario 1 & Scenario 2 & Scenario 1 & Scenario 2 \\
0.751 & 0.725 & 0.751 & 0.732 & 0.751 \\
\hline
\end{tabular}

The results in Table 1 are as expected. In the second scenario the OEE was the same as the reference state since the production maintained the normal schedule. But it is expected that it will affect other indicators because the data will later have to be asynchronously updated in the MES database. In the first scenario the production had to wait for the disruption to be solved, thus affecting the indicator. The impact is greater if the disruption lasts longer since, for shorter events, the connection is re-established before the current order is finished, causing no harm to the availability of the station. This observation shows that there are certain time intervals where the normal connection to the support system may be lost without affecting the OOE, which can be useful for maintenance purposes.

This paper presents a simulation-based methodology capable of evaluating the impacts of disruption events and finding suitable response actions to increase resilience in Cyber-Physical Production Systems processes. The methodology's focus is the use of a simulation tool to model the production system and the information systems, which simulates the communication between the two agents, the data flow, and the behaviour of the system during the disruptive event. For future research, the modelling of the communication between the manufacturing system and its information system should be more detailed and closer to a real OPC server.

\section{Acknowledgements}

This work is financed by National Funds through the Portuguese funding agency, FCT Fundação para a Ciência e a Tecnologia, within project UIDB/50014/2020.

\section{References}

[1] H. Panetto, B. Iung, D. Ivanov, G. Weichhart, and X. Wang, "Challenges for the 
cyber-physical manufacturing enterprises of the future," Annu. Rev. Control, vol. 47, pp. 200-213, 2019, doi: 10.1016/j.arcontrol.2019.02.002.

[2] N. Galaske, D. Strang, and R. Anderl, "Process deviations in cyber-physical production systems," Lect. Notes Eng. Comput. Sci., vol. 2220, no. October, pp. 1035-1040, 2015.

[3] J. Clausen, J. Larsen, A. Larsen, and J. Hansen, "Disruption Management: Operations Research between planning and execution," ORMS Today, vol. 28, no. 5, pp. 40-43, 2001.

[4] C. Schneeweiß, "Zur Bewältigung von Unsicherheiten in der Produktionsplanung und -steuerung," Lücke, Wolfgang Betriebswirtschaftliche Steuerungs- und Kontrollprobleme-wiss. Tagung d. Verb. d. Hochschullehrer für Betriebswirtschaft, no. e.V. an d. Univ. Göttingen. Wiesbaden, pp. 285-302, 1988.

[5] A. Ingemansson and G. S. Bolmsjö, "Improved efficiency with production disturbance reduction in manufacturing systems based on discrete-event simulation," J. Manuf. Technol. Manag., vol. 15, no. 3, pp. 267-279, 2004, doi: 10.1108/17410380410523498.

[6] K. Knüppel, G. Meyer, and P. Nyhuis, "A Universal Approach to Categorize Failures in Production," vol. 8, no. 2, pp. 240-243, 2014.

[7] N. Galaske and R. Anderl, "Disruption Management for Resilient Processes in Cyber-physical Production Systems," Procedia CIRP, vol. 50, pp. 442-447, 2016, doi: 10.1016/j.procir.2016.04.144.

[8] M. Moghaddam and A. Deshmukh, "Resilience of cyber-physical manufacturing control systems," Manuf. Lett., vol. 20, pp. 40-44, 2019, doi: 10.1016/j.mfglet.2019.05.002.

[9] N. Stricker and G. Lanza, "The concept of robustness in production systems and its correlation to disturbances," Procedia CIRP, vol. 19, no. C, pp. 87-92, 2014, doi: 10.1016/j.procir.2014.04.078.

[10] W. C. Wieland A, "Dealing with supply chain risks," Int. J. Phys. Distrib. Logist. Manag., vol. 42, no. 10, pp. 887-905, 2012.

[11] M. Heinicke, "Implementation of resilient production systems BY production control," Procedia CIRP, vol. 19, no. C, pp. 105-110, 2014, doi: 10.1016/j.procir.2014.05.001.

[12] E. Bernardes and M. Hanna, "A theoretical review of flexibility, agility and responsiveness in the operations management literature: Toward a conceptual definition of customer responsiveness," Int. J. Oper. Prod. Manag., vol. 29, no. 1, pp. 30-53, 2009.

[13] A. C. A. Cauvin, A. F. A. Ferrarini, and E. T. E. Tranvouez, "Disruption management in distributed enterprises: A multi-agent modelling and simulation of cooperative recovery behaviours," Int. J. Prod. Econ., vol. 122, no. 1, pp. 429439, 2009, doi: 10.1016/j.ijpe.2009.06.014.

[14] Hanser, "Methodenlehre der Betriebsorganisation," 1991.

[15] X. Gu, X. Jin, J. Ni, and Y. Koren, "Manufacturing system design for resilience," Procedia CIRP, vol. 36, pp. 135-140, 2015, doi: 10.1016/j.procir.2015.02.075.

[16] G. Murino, A. Armando, and A. Tacchella, "Resilience of Cyber-Physical Systems: An Experimental Appraisal of Quantitative Measures," Int. Conf. Cyber Conflict, CYCON, vol. 2019-May, no. 830892, pp. 1-19, 2019, doi: 10.23919/CYCON.2019.8757010. 